\title{
Board 15: Understanding Ambiguity in Engineering Problem Solving
}

\section{Dr. Elliot P. Douglas, University of Florida}

Elliot P. Douglas is Professor of Environmental Engineering Sciences,Associate Director for Research of the Institute for Excellence in Engineering Education, and Distinguished Teaching Scholar at the University of Florida. His research interests are in the areas of problem-solving, cultures of inclusion in engineering, engineering ethics, and environmental justice.

\section{Dr. David J Therriault, University of Florida}

Dr. Therriault, an Assistant Professor joined the College of Education at University of Florida in 2004. He received his undergraduate degree in psychology from the University of New Hampshire and his M. A. and Ph.D. in cognitive psychology from the University of Illinois at Chicago. Dr. Therriault's primary research interests include the representation of text in memory, comprehending time and space in language, the link between attention and intelligence, the use of perceptual symbols in language, and educational issues related to these topics.

\section{Marah B. Berry, University of Florida}

Marah Berry is a first year PhD student at the University of Florida studying Environmental Engineering. Her research focuses on ambiguity in problem solving. Her interest for problem solving began while she obtained her M.E. in Systems Engineering at the Pennsylvania State University. 


\title{
Understanding Ambiguity in Engineering Problem Solving
}

\begin{abstract}
Engineers are often faced with complex, unique, and challenging problems. Although a core activity of engineering is being able to solve complex problems efficiently and effectively, most engineering problems contain ambiguous elements. Engineers that are confident handling ambiguity are needed to solve real-world problems. Within the literature, engineering problems are typically characterized as either well-structured or ill-structured. Ambiguity, if it is mentioned at all in the problem characterization, goes undefined. Another issue is that ambiguity has only been identified as a structural element of the problem, ignoring how problem solvers may experience ambiguity differently in the same problem. Without a better understanding of ambiguity in problem solving, it is difficult to develop educational approaches that will teach students how to deal with ambiguity.

The goal of this project is to understand the different ways that students and practicing engineers experience ambiguity during problem solving. We aim to interview 20-30 senior civil engineering students and 20-30 practicing civil engineers on their experiences of ambiguity. Interviews will be conducted using artifact elicitation, in which each participant will bring a problem they have encountered which they consider to have been ambiguous. Interviews will be analyzed using phenomenography, leading to outcome spaces that define a hierarchy of ways that each group experiences ambiguity. These outcome spaces will then be used to develop a taxonomy of ambiguity that can be used in future studies of engineering problem solving. Ultimately, we aim to provide better instructional materials, methods, and tool kits for teaching students to solve ambiguous engineering problems.
\end{abstract}

\section{Introduction}

How do engineers handle ambiguous problems? This is a common question pondered by both academic and professional engineers. "Maturity of mind is the capacity to endure uncertainty," said John Huston Finley [1], former President of the College of the City of New York, Commissioner of the State of New York, and New York Times Editor-in-Chief. In contrast, in our previous research a senior engineering student stated "well basically that if you have the right equations then you can solve anything," [2] - [4]. These quotes provide contrasting interpretations of ambiguous problems. Finley implies that successfully navigating ambiguity is what makes someone an expert, whereas the student views ambiguity as nonexistent because all problems are solvable through equations.

Most discussions of engineering problem solving treat ambiguity as a characteristic of the problem. However, previous work, as represented by the quote above and Schrader et al. [5] indicate that ambiguity in the same problem may be experienced differently by problem solvers. Schrader et al. [5, p. 74] argue that "one core task in problem solving is the choice of the problem's uncertainty and ambiguity levels in the process of problem framing." Another issue is that many engineering problems are dichotomously characterized as well-structured (unambiguous) or ill-structured (ambiguous). However, we have found little research that 
specifies different types of ambiguity in a problem or possible individual differences in how ambiguity is experienced.

The goal of this project is to address the two issues described above: to help define the scope of ambiguity and examine ambiguity as at least partially originating in the problem solver during engineering problem solving. We aim to understand the different ways that students and practicing engineers experience ambiguity during problem solving. Developing a taxonomy of ambiguity will help to provide a deeper understanding of how to deal with ambiguity and how it is manifested in engineering problems. Understanding the processes that the problem solver goes through will aid us in developing better educational strategies and approaches to handling ambiguity. The research question for this study is: What are the qualitatively different ways that novice and expert civil engineers experience ambiguity? We are currently collecting data, so at this time we do not have any firm results. The purpose of this paper is to describe our plans.

\section{Literature Review}

A review of the literature shows that ambiguity has been discussed in relation to personal tolerance, but has not yet been defined within the context of engineering problem solving (although we recognize that other concepts, such as ill-structuredness, are related to ambiguity). Although, most engineering problems are characterized as ill-structured, ambiguous problems, it is difficult to understand the problem solvers' responses to ill-structured problems without a clear definition of ambiguity.

\section{Operationalizations of Ambiguity}

When determining how to frame our understanding of ambiguity we turned to its place in the psychology literature. Three distinct areas of ambiguity are discussed in the psychological literature: personality, the psychology of reading, and problem solving. One of the first mentions of ambiguity in the psychological literature is the work of Frenkle-Brunswik [6], [7] that discusses intolerance of ambiguity. Understanding how a person reacts to an ambiguous situation can be helpful when assessing personality [7]. Frenkle-Brunswik [7] demonstrated that children who are more rigid in their responses to ambiguous stimuli tend to have a more ethnocentric personality and demonstrate authoritarian characteristics. They also tend to reject the unusual, need order, and lack the understanding of how good and bad can exist in one person. In the early literature, the term ambiguity became synonymous with the terms authoritarian and prejudice [8].

The psychology of reading is another area of research that that operationalizes ambiguity [9] [11]. Reading comprehension theories help to explain how readers handle ambiguity found in text. An example of ambiguity while reading is a word that has multiple possible meanings i.e. I am going to the bank. Is the bank characterized as a river or a financial institution? Ambiguity in language is typically categorized as either lexical (previous example) or syntactic, where sentence structures can have multiple possibilities and meanings. Many studies have concluded that readers attribute multiple meanings to words in text [12]. The level of activation of the correct interpretation is dependent on the frequency and context [13], and the process works similarly for sentence structure [14]. 
Surprisingly, ambiguity is rarely operationalized with respect to problem solving. Schrader, Riggs, and Smith [5], to our knowledge, are some of the only researchers to try to operationalize uncertainty and ambiguity with respect to problem solvers. They argue that ambiguity and uncertainty are two distinct terms. Uncertainty is categorized as lacking information whereas ambiguity is categorized as lack of clarity of relationships. Reducing uncertainty can be done by gathering information and building models, and problem framing can help to diminish ambiguity [5]. Schrader, et al. [5] continue to argue that the problem solver determines the level of uncertainty and ambiguity when they frame the problem space. For example, if the solver has previous knowledge in a similar problem space then ambiguity could be reduced.

\section{Engineering Problem Solving}

Although engineering problems are frequently recognized as ambiguous, there is very little literature that defines what it means for an engineering problem to be "ambiguous". Professional engineers solve ill-structured, complex problems daily. These problems typically have multiple paths to the correct answer. How to reach the correct answer is dependent on the development of the problem space and how the individual frames the question. Developing skills to solve these complex ambiguous problems is required from students, educators, and professional engineers. There has been a significant amount of research on what types of problems are solved by engineers [15] - [20], but they primarily focus on the nature of the problems and not on how one makes choices in assessing ambiguity while solving them [21].

Engineering problems are typically characterized as open-ended or ill-structured. Research has shown that well-structured problems are simple, concrete, and have a single solution whereas illstructured problems are complex, abstract, and have multiple possible solutions [22], [23]. Jonassen [19], [23] and Jonassen and Hung [24] developed the most well-known typology of problems. The classification of problems included eleven categories when it was first developed and was later modified to include ten categories. The problem categories range from algorithmic to dilemma. Another group of typologies comes from Johnston [25], Reid and Yang [26], and Simon [27] which describe problems from defined to highly ambiguous. While this research provides some guidance with respect to possible types of problem ambiguity, these typologies fail to describe the role of the problem solver in attempting the problem. Other work has indicated that problem-solving strategies were related to students' epistemic beliefs [2] and that students see a strong disconnect between academic and workplace problems [3], [4].

Current research on how epistemological beliefs affect problem solving does not address the question of how the problem solver chooses ambiguity. Instead these studies assume that problems have a certain characteristic (e.g. well- vs. ill-structured) and examine the relationship between epistemological beliefs and problem solving capabilities. Epistemological beliefs and problem solving have been studied across several disciplines in a variety of ways including engineering, physics, chemistry, and mathematics [28] - [34]. The results from these studies have varied from finding no relationship to finding some significant relation between ill-structured problem solving and epistemological beliefs. Most of these studies focus on the characteristics of the needs of the problems, ignoring the role of the problem solver. Faber and Benson [35] studied how students' epistemological beliefs affect their problem-solving goals. They found that 
that the instructional context in which the problem was presented resulted in some students treating an open-ended problem as if it were closed-ended with a single correct answer.

\section{Novice- Expert Differences in Problem Solving}

Novice and experts experience and process information differently when solving problems [36] [38]. Chi, et al. [36] and Ericsson [39], [40] argue that experts rely on large bodies of knowledge relating to their skills in that specific discipline. These skills do not always transfer directly to other domains. Experts know more about their domains than novices. This therefore allows them to perform tasks in qualitatively different ways. For example, Larkin et al. [37] claimed that experts have a complex schema of possible solution guides and paths to solving a problem which are developed over time. The research on engineering problem solving has focused on design problems. Several papers from Atman and colleagues examined problem solving with expert and novices by using the think aloud methodology [41] - [46]. The results demonstrated that quality of design increased with experience. Experts were also able to articulate their process more easily. Ahmed, Wallace, and Blessing [15] found similar results when using an ethnographic approach.

\section{Methodology}

This study will use phenomenography to define different ways that engineering problem solvers experience ambiguity. Marton [47] described phenomenography as bringing together research on variation across multiple domains and topics under one umbrella. The result is an outcome space defining the variation in the way something can be experienced. In the sections below, we describe the anticipated theoretical considerations, sampling, data collection, and analysis approach.

\section{Theoretical Considerations}

In the current conception of phenomenography there are two important considerations: its epistemological assumptions and the use of variation theory to explain how something can be experienced differently, although these aspects are intimately related to one another. Variation theory describes how the different aspects of a phenomenon are experienced only when there is a perceived variation in that phenomenon. Bussey et al. [48] discussed the example of a ripening banana as an example of phenomenography. We experience bananas ripening when the color changes from green to yellow and eventually to brown. If the banana is peeled, it is no longer evident if the banana is ripening because the peel is removed, so no color variation is experienced. Thus, "[t]he limited number of features to which one attends and the meaning one ascribes to those features will determine an individual's perception of a given event [48, p. 9]."

The variations in an experience is focused on in phenomenography by viewing the description of the nature of the experience [49]. The categories that are developed from the experiences are the results of a phenomenographic analysis and explain the variety of ways that an event can be experienced by a group of individuals [50]. Individuals can experience a phenomenon in multiple ways. In the banana example, the categories of ripeness may be color, taste, and smell. 
Depending on what feature the individuals attends to will determine which category is salient depending on how the banana is presented.

The underlying epistemology of phenomenography is non-dualism, which is described by Pang [49] and Sjostrom \& Dahlgren [51] as the meaning made through an individual's relationship with the world. Meaning is made through variation and that variation only exists when one has assigned meaning to both an experience as separate from its context (what Pang [49] calls the referential aspect) and the internal structure of the experience. "Structure presupposes meaning and meaning presupposes structure. Structure and meaning thus mutually contribute to each other in the act of experiencing [49, p. 149]."

Bussey et al. [48] expands on Pang's [49] description by providing a further explanation of what features of variation lead to meaning-making. The three elements described are awareness, discernment, and simultaneity. Awareness is that aspect of the phenomenon that someone is aware of. If someone is not aware of some aspect, even if there is variation, that aspect will not become part of the phenomenon as experienced. Discernment is the ability to focus on that aspect and be attentive to its variation. Finally, to fully make meaning one must be aware of multiple varying aspects simultaneously. A single feature is insufficient.

\section{Sampling}

Participants are being recruited from the civil engineering domain. Phenomenography requires that the sample be relatively uniform. If there is an extensive amount of variation in the sample, then data synthesis will not be possible to develop outcome spaces. Focusing on the group of civil engineers is both a theoretical and pragmatic decision. From a theoretical perspective, civil engineers work on complex, ill-structured problems in both academics and professionally. Many of the problems also have a social focus (i.e., how will changing the stoplight pattern increase commuting of drivers during rush hour?). From a pragmatic perspective, the researchers are in an academic unit that includes civil engineering, making it possible to gain access to the student population.

We are aiming to interview 20-30 practicing civil engineers and 20-30 civil engineering students. For the phenomenographic analysis the samples will be treated as two individual sets due to the expected large variation in approaches between novices and experts. The students will be recruited from senior-level classes. Senior-level students are chosen because they will have more experience than the underclassmen and have experienced several different problem types. We will not place restrictions on participation in relation to ability (i.e., grade point average). We will oversample both women and underrepresented minorities. The practicing engineers will be recruited through the department's industrial advisory board and alumni list. We will apply the restriction that the professionals have at least ten years of experience. We will also oversample the professional engineers for women and underrepresented minorities. Interviews for both samples will be held in person when possible and using videoconferencing when not. 


\section{Artifact Elicitation Interviews}

The interviews will be conducted using a phenomenographic semi-structured approach in combination with artifact elicitation [52]. Photo elicitation and artifact elicitation are similar techniques and help to provide a means to begin by providing a concrete artifact to ground discussion. It also helps the interviewee to clearly describe how they approached a certain problem or process.

It is expected that the interviews will last around an hour, but no time limit will be placed on them. The participants will be asked to bring two or three examples of problems they have worked on that they felt had ambiguity. The interview will start by eliciting from the participant what aspects of the problem were ambiguous and why they felt the problem was ambiguous. Probing questions will be asked as needed. Some potential probing questions include the following:

- What other projects have you worked on that had some aspect that was ambiguous?

- What do you typically do when you are confronted with ambiguity?

- What are the different types of ambiguity that you have experienced?

- What are the differences between an ambiguous and unambiguous problem?

Remaining consistent with phenomenography and variation theory, interviews will focus on the different ways participants have experienced ambiguity. We will purposefully ask questions to highlight the variations of ambiguity experienced. For example, we will inquire about what makes a problem ambiguous or unambiguous, as well as what information would change the level of ambiguity. We will inquire about what types of ambiguity are more difficult to deal with than others and why.

\section{Data Analysis}

Our analysis will follow the approach developed by Marton [50] and Frank [53]. A basic overview of this approach is to become familiar with the data, develop an initial draft of the outcome space, refine the outcome space, determine the categories each transcript represents, refine the draft of the outcome space, then finalize the outcome space [53].

In phenomenography, the unit of analysis is considered to be the transcript. The interviews will be transcribed verbatim and will be read multiple times. This reading will allow us to holistically understand how each participant conceived ambiguity. The experiences of ambiguity that are defined in the interviews will be identified. Quotes from the participants will be placed in a data pool that will be analyzed holistically from the entire group. Akerlind [54] suggests that phenomenography considers the range of understandings within an entire group rather than one individual's perspective.

Emergent categories will be used to sort the quotes into the different ways that ambiguity was experienced. The transcripts will then be reexamined holistically to identify the way the categories are represented. If there needs to be a modification of the categories during this part of 
the analysis it will be documented and updated. Identifying logical relationships between the categories is also part of the process. When using phenomenography, it is assumed that there are levels of sophistication. In any given experience one needs to experience the less sophisticated levels before moving on to the more complex sophisticated ones [55].

The result of the analysis will be an outcome space, which is a pictorial diagram that displays the hierarchy of the qualitatively different ways that one experiences ambiguity. The transcripts for novice and experts will be analyzed as two different outcome spaces. If enough commonalities can be readily identified then there is a possibility of combining the outcome spaces into one. The categories identified through the analysis will be become the taxonomy of ambiguity.

\section{Quality Considerations}

To ensure we have a quality study, we have identified potential threats to the research and what steps will be taken to mitigate them. One potential threat is that there will be a lack of convergence due to differing experiences with ambiguity. The analysis procedure has been developed with this potential threat in mind. Using artifact elicitation as part of the procedure can lead to potential convergence issues due to the participants bringing a large array of different engineering problems. If this happens it could lead to an interesting result of how ambiguity can be experienced across an array of different problems, but this would limit the possibilities of being able to develop an outcome space. There will be a potential modification of the types of artifacts that are elicited if the pilot interviews display this threat. The protocol can also be modified to focus on ambiguity in certain settings.

A second potential threat is the difficulty in bracketing the experience of the two groups. The analysis for each group could be done in parallel or sequentially. If it is analyzed in parallel, it will be hard to immerse completely into both datasets at the same time. If it is done sequentially it may be difficult to not allow one dataset's categories to influence the others. We believe for this study it is most appropriate to use a sequential approach. Conversations within the research team throughout the analysis will provide a check to ensure appropriate bracketing.

The quality of the project will be reviewed both internally and externally. Externally the quality will be monitored by an advisory board and internally it will be monitored by the usage of the $\mathrm{Q}^{3}$ framework [56], [57]. The $\mathrm{Q}^{3}$ framework focuses on the data collection and data handling processes. We will use this framework as a set of guiding questions rather than a checklist. The framework focuses on five area for validation: theoretical, procedural, communicative, pragmatic, and ethical, as well as process reliability. It then has a series of questions that researchers can use as a guide. For example, under process reliability it asks "How can we capture and record the constructions of participants' social realities in a dependable way?" We will revisit the $\mathrm{Q}^{3}$ framework in the beginning of the project and throughout as the project progresses. We will modify the project protocol as needed when reviewing the $\mathrm{Q}^{3}$ framework for quality. 


\section{Conclusion}

The literature review illustrates that ambiguity has not been adequately operationalized. Our project has just begun, so at this time we are collecting data, but the goal of this project is to understand the different ways that students and practicing engineers experience ambiguity during problem solving. The result of our project will be a taxonomy of ambiguity developed from the outcome space(s). This taxonomy will deepen our understanding of problem solving, allowing us and other researchers to further explore the role of ambiguity. For example, the taxonomy could be used to develop a series of problems that illustrate different types of ambiguity that are experienced by engineers. We can then study the relationships between ambiguity and other factors such as domain knowledge, working memory, epistemological beliefs, etc. Understanding these different aspects of ambiguity will help to reveal potential relationships with cognitive and affective behaviors that affect learning. We will also seek to understand how students respond to various levels of ambiguity before developing educational interventions. Ultimately, we aim to provide better instructional materials, methods, and tool kits for teaching students to solve ambiguous engineering problems.

\section{Acknowledgement}

Funding was provided by National Science Foundation grant EEC-1824610.

\section{References}

[1] J. Finley Library of Quotes. [Online]. Available: http://www.libraryofquotes.com/. Accessed Tue 16 Jan 18], n.d.

[2] E. P. Douglas, M. Koro-Ljungberg, N. J. Mcneill, Z. T. Malcolm, and D. J. Therriault, "Moving beyond formulas and fixations: solving open-ended engineering problems," European Journal of Engineering Education, vol. 37, no. 6, pp. 627-651, 2012.

[3] M. Koro-Ljungberg, E. P. Douglas, N. J. McNeill, D. J. Therriault, C. S. Lee, and Z. Malcolm, "Academic problem-solving and students' identities as engineers," The Qualitative Report, vol. 22, no. 2, pp. 456-478, 2017.

[4] N. J. Mcneill, E. P. Douglas, M. Koro-Ljungberg, D. J. Therriault, and I. Krause, "Undergraduate Students Beliefs about Engineering Problem Solving," Journal of Engineering Education, vol. 105, no. 4, pp. 560-584, 2016.

[5] S. Schrader, W. M. Riggs, and R. P. Smith, "Choice over uncertainty and ambiguity in technical problem solving," Journal of Engineering and Technology Management, vol. 10, no. 1-2, pp. 73-99, 1993.

[6] E. Frenkel-Brunswik, "A Study of Prejudice in Children," Human Relations, vol. 1, no. 3, pp. 295-306, 1948.

[7] E. Frenkel-Brunswik, "Intolerance Of Ambiguity As An Emotional And Perceptual

Personality Variable,” Journal of Personality, vol. 18, no. 1, pp. 108-143, 1949. 
[8] A. Furnham and J. Marks, "Tolerance of Ambiguity: A Review of the Recent Literature," Psychology, vol. 04, no. 09, pp. 717-728, 2013.

[9] W. Kintsch, Comprehension: a paradigm for cognition. Cambridge: Cambridge University Press, 1998.

[10] K. Rayner, A. Pollatsek, J. Ashby, and C. Clifton Jr, Psychology of reading. Ne York: Psychology Press, 2012.

[11] T. A. van. Dijk and W. Kintsch, Strategies of discourse comprehension. London: Academic Press, 1983.

[12] D. A. Swinney, "Lexical access during sentence comprehension: (Re)consideration of context effects," Journal of Verbal Learning and Verbal Behavior, vol. 18, no. 6, pp. 645-659, 1979.

[13] S. A. Duffy, R. K. Morris, and K. Rayner, "Lexical ambiguity and fixation times in reading," Journal of Memory and Language, vol. 27, no. 4, pp. 429-446, 1988.

[14] M. C. Macdonald, N. J. Pearlmutter, and M. S. Seidenberg, "The lexical nature of syntactic ambiguity resolution.," Psychological Review, vol. 101, no. 4, pp. 676-703, 1994.

[15] S. Ahmed, K. M. Wallace, and L. T. Blessing, "Understanding the differences between how novice and experienced designers approach design tasks," Research in Engineering Design, vol. 14, no. 1, pp. 1-11, 2003.

[16] B. T. Christensen and C. D. Schunn, "The relationship of analogical distance to analogical function and preinventive structure: the case of engineering design," Memory \& Cognition, vol. 35, no. 1, pp. 29-38, 2007.

[17] R. Fernandes and H.A. Simon, "A study of how individuals solve complex and illstructured problems," Policy Sciences, vol. 32, no. 3, pp. 225-244, 1999.

[18] P. Kumsaikaew, J. Jackman, and V. J. Dark, "Task Relevant Information in Engineering Problem Solving," Journal of Engineering Education, vol. 95, no. 3, pp. 227-239, 2006.

[19] D. H. Jonassen, "Engineers as Problem Solvers," Cambridge Handbook of Engineering Education Research, pp. 103-118.

[20] D. Jonassen, J. Strobel, and C. B. Lee, "Everyday Problem Solving in Engineering: Lessons for Engineering Educators," Journal of Engineering Education, vol. 95, no. 2, pp. 139-151, 2006.

[21] D. H. Jonassen, “A taxonomy of meaningful learning,” Educational Technology, vol 47, no.5, pp. 30-35, 2007.

[22] D. H. Jonassen, "Instructional design models for well-structured and III-structured problem-solving learning outcomes," Educational Technology Research and Development, vol. 45, no. 1, pp. 65-94, 1997.

[23] D. H. Jonassen, "Toward a design theory of problem solving," Educational Technology Research and Development, vol. 48, no. 4, pp. 63-85, 2000.

[24] D. H. Jonassen and W. Hung, "All Problems are Not Equal: Implications for ProblemBased Learning," Interdisciplinary Journal of Problem-Based Learning, vol. 2, no. 2, pp. 6-28, 2008.

[25] A. H. Johnstone, "Can problem solving be taught," University Chemistry Education, vol. 5, no. 2, 69-73, 2001. 
[26] N. Reid and M.-J. Yang, "Open-ended problem solving in school chemistry: A preliminary investigation," International Journal of Science Education, vol. 24, no. 12, pp. 1313-1332, 2002.

[27] H. A. Simon, "The structure of ill structured problems," Artificial Intelligence, vol. 4, no. 3-4, pp. 181-201, 1973.

[28] C. Angeli and N. Valanides, "Epistemological beliefs and ill-structured problem-solving in solo and paired contexts," Educational Technology \& Society, vol 15, no. 1, pp. 2-14, 2012.

[29] A. R. P. de Ataide and I. M. Greca, "Epistemic views of the relationship between physics and mathematics: Its influence on the approach of undergraduate students to problem solving," Science \& Education, vol 22, no. 6, pp. 1405-1421, 2013.

[30] E. P. Douglas, M. Koro-Ljungberg, D. J. Therriault., C. S. Lee, N. McNeill, and Z. T. Malcolm, Discourses and social worlds in engineering education: Preparing problemsolvers for engineering practice, Paper presented at the American Society for Engineering Education Annual Conference, San Antonio, TX, 2012.

[31] V. Mehdinezhad and Z. Bamari, "The Relationship between Test Anxiety, Epistemological Beliefs and Problem Solving among Students," Journal of New Approaches in Educational Research, vol. 4, no. 1, pp. 2-8, 2015.

[32] K. R. Muis, C. Psaradellis, S. P. Lajoie, I. Di Leo, and M. Chevrier, "The role of epistemic emotions in mathematics problem solving," Contemporary Educational Psychology, vol 42 , pp. 172-185, 2015.

[33] G. Schraw, M. E. Dunkle, and L. D. Bendixen, "Cognitive processes in well-defined and ill-defined problem solving," Applied Cognitive Psychology, vol. 9, no. 6, pp. 523-538, 1995.

[34] D. Stamovlasis, "Methodological and epistemological issues on linear regression applied to psychometric variables in problem solving: rethinking variance," Chem. Educ. Res. Pract., vol. 11, no. 1, pp. 59-68, 2010.

[35] C. Faber and L. C. Benson, "Engineering Students Epistemic Cognition in the Context of Problem Solving," Journal of Engineering Education, vol. 106, no. 4, pp. 677-709, 2017.

[36] M. T. Chi, P. J. Feltovich, and R. Glaser, "Categorization and representation of physics problems by experts and novices," Cognitive science, vol 5, no. 2 , pp. 121-152, 1981.

[37] J. H. Larkin, J. McDermott, D. P. Simon, and H. A. Simon, "Expert and novice performance in solving physics problems," Science, vol 208, no. 4450, pp. 1335-1342, 1980.

[38] J. H. Larkin, “Display-based problem solving,” In D. Klahr \& K. Kotovsky (Eds.), Complex information processing: The impact of Herbert A. Simon, pp. 319-341, 1989.

[39] K. A. Ericsson, The road to excellence: The acquisition of expert performance in the arts and sciences, sports, and games. Psychology Press, 2014.

[40] K. A. Ericsson, "Expertise and individual differences: the search for the structure and acquisition of experts' superior performance," Wiley Interdisciplinary Reviews: Cognitive Science, vol 8, no. 1-2, 2017.

[41] C. J. Atman, R. S. Adams, M. E. Cardella, J. Turns, S. Mosborg, and J. Saleem, "Engineering design processes: A comparison of students and expert practitioners," Journal of Engineering Education, vol 96, no. 4, pp. 359-379, 2007. 
[42] C. J. Atman and K. M. Bursic, "Teaching engineering design: Can reading a textbook make a difference?" Research in Engineering Design-Theory Applications and Concurrent Engineering, vol 8, no . 4, pp. 240-250, 1996.

[43] C. J. Atman and K. M. Bursic, "Verbal protocol analysis as a method to document engineering student design processes," Journal of Engineering Education, vol 87, pp. 121-132, 1998.

[44] C. J. Atman, M. E. Cardella, J. Turns, and R. Adams, "Comparing freshman and senior engineering design processes: An in-depth follow-up study," Design Studies, vol 26, no. 4, pp. 325-357, 2005.

[45] C. J. Atman, J. R. Chimka, K. M Bursic, and H. L. Nachtmann, “A comparison of freshman and senior engineering design processes," Design Studies, vol 20, no. 2, pp. 131-152, 1999.

[46] C. J. Atman, D. Kilgore, and A. McKenna, "Characterizing design learning: A mixedmethods study of engineering designers' use of language," Journal of Engineering Education, vol 97, no. 3, pp. 309-326, 2008.

[47] F. Marton, "Phenomenography - describing conceptions of the world around us," Instructional Science, vol. 10, pp. 177-200, 1981.

[48] T. J. Bussey, M. Orgill, and K. J. Crippen, "Variation theory: A theory of learning and a useful theoretical framework for chemical education research," Chemistry Education Research and Practice, vol 14, pp. 9-22, 2013.

[49] M. F. Pang, "Two faces of variation: On continuity in the phenomenographic movement," Scandinavian Journal of Educational Research, vol 47, no. 2, pp. 145-156, 2003.

[50] F. Marton, "Phenomenography — a research approach to investigating different understandings of reality," Journal of Thought, vol 21, no. 3, pp. 28-49, 1986.

[51] B. Sjostrom, and L. O. Dahlgren, "Applying phenomenography in nursing research," Journal of Advanced Nursing, vol 40, no. 3, pp. 339-345, 2002.

[52] E. P. Douglas, S. S. Jordan, M. Lande, and A. E. Bumbaco, Artifact elicitation as a method of qualitative inquiry in engineering education. Paper presented at the ASEE Annual Conference, Seattle, WA, 2015.

[53] D. Z. Frank, Investigating culturally-contextualized making with the Navajo Nation, (PhD), University of Florida, 2018.

[54] G. S. Akerlind, "Variation and commonality in phenomenographic research methods," Akerlind, Gerlese S., vol 24, no. 4, pp. 321-334, 2005.

[55] M. Micari, G. Light, S. Calkins, and B. Streitwieser, "Assessment beyond performance," Phenomenography in educational evaluation. American Journal of Evaluation, vol 28, no 4, pp. 458-476, 2007.

[56] J. Walther, N. W. Sochacka, L. C. Benson, A. E. Bumbaco, N. Kellam, A. L. Pawley, and C. M. L. Phillips, "Qualitative research quality: A collaborative inquiry across multiple methodological perspectives," Journal of Engineering Education, vol 106, no. 3, pp. 398-430, 2017.

[57] J. Walther, N. W. Sochacka, and N. N. Kellam, "Quality in interpretive engineering education research: Reflections on an example study," Journal of Engineering Education, vol 102, no. 4, pp. 626-659, 2013. 